International Journal of Pure and Applied Mathematics

Volume 83 No. 3 2013, 507-514

ISSN: 1311-8080 (printed version); ISSN: 1314-3395 (on-line version)

url: http://www.ijpam.eu

doi: http://dx.doi.org/10.12732/ijpam.v83i3.11

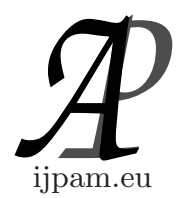

\title{
SUBALGEBRAS AND QUOTIENT ALGEBRAS OF PARAFREE LIE ALGEBRAS
}

\author{
Zehra Velioğlu \\ Department of Mathematics \\ Cukurova University \\ Adana, TURKEY
}

\begin{abstract}
We prove that any subalgebra and any quotient algebra of a parafree Lie algebra are parafree.
\end{abstract}

AMS Subject Classification: 17B99, 17B01

Key Words: Lie algebras, Parafree subalgebra, quotient algebra

\section{Introduction}

Current interest in parafree Lie algebras arose from works of Gilbert Baumslag ([2],[3], [4],[5],[6]). In $1978 \mathrm{H}$. Baur has translated the notion of parafree groups to parafree Lie algebras $([8],[9])$. There are some difficulties in the translation procedure from groups to Lie algebras. These difficulties are pointed out by Baur [9].Works of Baur [9] and Knus and Stammbach [12] are the subject matter of this paper. In this work we turn our attention to the subalgebra structure of parafree Lie algebras. Our purpose is to investigate the properties of subalgebras of parafree Lie algebras and quotient algebras. Further we demonstrate that if a parafree Lie algebra has the same rank as its quotient subalgebra, then the divisor ideal is trivial.

Received: November 19, 2012

(c) 2013 Academic Publications, Ltd. url: www.acadpubl.eu 


\section{Preliminaries}

Let $L$ be a Lie algebras over a field $k$. The lower central series

$$
L=\gamma_{1}(L) \supseteq \gamma_{2}(L) \supseteq \cdots \supseteq \gamma_{n}(L) \supseteq \cdots
$$

is defined inductively by

$$
\gamma_{2}(L)=L^{\prime}=[L, L], \gamma_{n+1}(L)=\left[\gamma_{n}(L), L\right], n \geq 1
$$

If $n$ is the smallest integer satisfying $\gamma_{n}(L)=0$ then $L$ is called nilpotent of degree $n$.

Definition 1. A Lie algebra $L$ is called residually nilpotent if ,

$$
\bigcap_{n=1}^{\infty} \gamma_{n}(L)=\{0\}
$$

equivalently, given any non-trivial element $u \in L$, there exists an ideal $J$ of $L$ such that $u \notin J$ with $L / J$ nilpotent. We define here the notion of parafree Lie algebras.

We associate with the lower central series of L its lower central sequence:

$$
L / \gamma_{2}(L), L / \gamma_{3}(L), \ldots
$$

We say that two Lie algebras $\mathrm{L}$ and $\mathrm{H}$ have the same lower central sequence if $L / \gamma_{n}(L) \cong H / \gamma_{n}(H)$ for every $n \geq 1$.

Definition 2. The Lie algebra $L$ is called parafree over a set $X$ if,

i $L$ is residually nilpotent, and

ii $L$ has the same lower central sequence as a free Lie algebra generated by the set $X$.

The cardinality of $\mathrm{X}$ is called the rank of $\mathrm{L}$.

A paragenerating set for $L$ is the set $X \subset L$ which freely generates $L$ modulo $\gamma_{2}(L)$. 


\section{Some Useful Results}

Theorem 3. A finitely generated m-generator Lie algebra with the same lower central sequence as a free Lie algebra of rank $m$ is free.

Proof. Let $L$ be an $m$-generator Lie algebra with the same lower central sequence as $F$, free Lie algebra of rank $m$, and suppose that $L$ is not free. Then the canonical mapping $\phi: F \rightarrow L$ which takes generators to generators is an epimorphism with nontrivial kernel; i.e., there is a word $w \in F, w \neq 0$ such that $\phi(w)=0$.

Since $F$ is residually nilpotent, there is a positive integer n such that $w \in$ $\gamma_{n}(F)$, but $w \notin \gamma_{n+1}(F)$. The verbal $\gamma_{n+1}(F)$ is mapped by $\phi$ onto $\gamma_{n+1}(H)$, so there is an induced epimorphism

$$
\bar{\phi}: F / \gamma_{n}(F) \rightarrow H / \gamma_{n}(H)
$$

with $\bar{\phi}: \bar{w} \rightarrow \overline{0}$; since $w \notin \gamma_{n+1}(F), \bar{\phi}$ is a mapping with a nontrivial kernel of a finitely generated nilpotent Lie algebra onto an isomorphic image of itself. This gives us the contradiction, because finitely generated nilpotent Lie algebras hopfian [11]. Thus $L$ is free.

Let $V$ is a variety whose free groups are residually nilpotent. M. Dean [10] prove that a set $\mathrm{X}$ which is a parabasis for a parafree- $\mathrm{V}$ group freely generates a free $\mathrm{V}$-group.

There is a corresponding theorem for parafree Lie algebras. The proof of the following theorem is the same as in the parafree groups case [10].

Theorem 4. Let $P$ be a parafree Lie algebra and $X$ is a paragenerating set of $P$. Then $X$ freely generates a free Lie algebra.

Proof. Let $H$ be the subalgebra of $P$ which is generated by $X$ and let $F$ be the free Lie algebra freely generated by a set $Y$, where $|X|=|Y|$. We are going to prove that

$$
H / \gamma_{n}(H) \cong F / \gamma_{n}(F),
$$

where $n \geq 1$ then by the Theorem 3 the subalgebra $\mathrm{H}$ will be free.Consider the epimorphism $\phi: Y \rightarrow X$ defined as

$$
\phi: y_{i} \rightarrow x_{i}
$$

where $y_{i} \in Y$ and $x_{i} \in X, 1 \leq i \leq|X|$. Then $\phi$ can be extended to a homomorphism $\bar{\phi}: F \rightarrow H$. 
Now we define the mappings

$$
\phi_{n}: F / \gamma_{n}(F) \rightarrow H / \gamma_{n}(H)
$$

as

$$
\phi_{n}: f+\gamma_{n}(F) \rightarrow \bar{\phi}(f)+\gamma_{n}(H)
$$

where $n \geq 1$.

Case 1: $|X|=|Y|<\infty$.

We need to prove that $\left(H+\gamma_{n}(P)\right) / \gamma_{n}(P)=P / \gamma_{n}(P)$.

Since $X$ freely generates $P$ modulo $\gamma_{2}(P)$ then it generates a free Lie algebra By the Theorem 9 of [1, section 4.2.2.] $X$ freely generates $H$ modulo $\gamma_{n}(P)$. Hence

$$
\left(H+\gamma_{n}(P)\right) / \gamma_{n}(P)=P / \gamma_{n}(P)
$$

By the Second Isomorphism Theorem,then we have

$$
H /\left(H \cap \gamma_{n}(P)\right)=P / \gamma_{n}(P)
$$

Clearly $H \cap \gamma_{n}(P) \supset \gamma_{n}(H)$, so there is a natural homomorphism from $H / \gamma_{n}(H)$ onto $H /\left(H \cap \gamma_{n}(P)\right)$ for $n=2,3, \ldots$ Consider the following diagram, which holds for $n=2,3, \ldots$

$$
F / \gamma_{n}(F) \underset{\bar{\phi}_{\mathrm{n}}}{\rightarrow} H / \gamma_{n}(H) \rightarrow H /\left(H \cap \gamma_{n}(P)\right) \cong P / \gamma_{n}(P)
$$

The diagram shows a homomorphism of $F / \gamma_{n}(F)$ onto an isomorphic copy of itself. Since finitely generated nilpotent Lie algebras are Hopfian [11], the kernel of this homomorphism is trivial; hence $\bar{\phi}$ is an isomorphism for $n=$ $2,3, \ldots$ By Theorem $3, H$ is freely generated by $X$.

Case 2: $|X|=|Y|=\infty$. Suppose $w \in \operatorname{ker} \phi(\phi: F \rightarrow H$, as in the Case $1)$. Let $F_{s}$ be a finitely generated subalgebra of $F$, freely generated by a subset $S \subseteq Y$, such that $w \in F_{s}$. Consider the smallest ideal $H_{s}$ of $P$ which generated by $\phi(S)$.

Let $\psi_{n}: F / \gamma_{n}(F) \rightarrow P / \gamma_{n}(P)$ be the family of isomorphism between the lower central sequence of $F$ and the lower central sequence of $P$.

Now, for $n=2,3, \ldots$, we have

$$
F_{s} / \gamma_{n}\left(F_{s}\right)=\left(F_{s}+\gamma_{n}(F)\right) / \gamma_{n}(F) \subseteq F / \gamma_{n}(F)
$$

Let $P_{s} / \gamma_{n}\left(P_{s}\right)$ denote the image $\psi_{n}\left(F_{s} / \gamma_{n}\left(F_{s}\right)\right)$. 
As before, we have $H_{s} /\left(H_{s} \cap \gamma_{n}\left(P_{s}\right)\right) \cong P_{s} / \gamma_{n}\left(P_{s}\right)$ and the following diagram, by hopficity, demonstrates that $F_{s} / \gamma_{n}\left(F_{s}\right) \cong H_{s} / \gamma_{n}\left(H_{s}\right)$ :

$$
F_{s} / \gamma_{n}\left(F_{s}\right) \rightarrow H_{s} / \gamma_{n}\left(H_{s}\right) \rightarrow H / H_{s} \cap \gamma_{n}(P) \cong P_{s} / \gamma_{n}\left(P_{s}\right)
$$

Again by Theorem 3, $H_{s}$ is free and $\phi$ restricted to $F_{s}$ is an isomorphism. Therefore, $w=0$, so $\phi$ is an isomorphism as well, and $H$ is free.

Corollary 5. Let $H$ be the subalgebra of $P$ defined in the Theorem 4 . Then, for $n=2,3, \ldots$

$$
\gamma_{n}(H)=H \cap \gamma_{n}(P) .
$$

\section{Subalgebras and Quotient Algebras}

Theorem 6. Any subalgebra of a parafree Lie algebra is again parafree.

Proof. Let $P$ be a parafree Lie algebra with the same lower central sequence as a free Lie algebra $F$. Let

$$
\phi: P \rightarrow F
$$

be the canonical mapping. Then $\phi$ induces the isomorphisms

$$
\phi_{n}: P / \gamma_{n}(P) \rightarrow F / \gamma_{n}(F)
$$

where $n \geq 2$.

Let $\mathrm{H}$ be an subalgebra of P. By the Corollary 5, we have $H \cap \gamma_{n}(P)=$ $\gamma_{n}(H)$, then $\bigcap_{n=1}^{\infty} \gamma_{n}(H) \subset \bigcap_{n=1}^{\infty} \gamma_{n}(P)$. Since $\mathrm{P}$ is parafree,then it is residually nilpotent, i.e. $\bigcap_{n=1}^{\infty} \gamma_{n}(P)=\{0\}$. Thus, we have

$$
\bigcap_{n=1}^{\infty} \gamma_{n}(H)=\{0\}
$$

That is, $\mathrm{H}$ is residually nilpotent.To prove parafreeness of $H$, we need to prove that $H$ has the same lower central sequence as a free Lie algebra. Let $K=\phi(H)$

Since every subalgebra of a free Lie algebra is free, then the subalgebra $K$ is free. Define mapping $\varphi$ as;

$$
\varphi: H \rightarrow K / \gamma_{n}(K)
$$




$$
x \rightarrow \phi(x)+\gamma_{n}(K)
$$

where $x \in H$.

Clearly $\varphi$ is Lie algebra epimorphism. Now we calculate the kernel of $\varphi$.

Let $x$ be an element of $\operatorname{ker} \varphi$, then $\varphi(x)=\phi(x)+\gamma_{n}(K)=\gamma_{n}(K)$.Therefore $\phi(x) \in \gamma_{n}(K) \subset \gamma_{n}(F)$. Clearly, $H \subseteq P$, then $x \in P$. By the definition of $\phi_{n}$

$$
\phi_{n}\left(x+\gamma_{n}(P)\right)=\phi(x)+\gamma_{n}(F) \text { and } \phi(x) \in \gamma_{n}(K)
$$

then $\phi_{n}\left(x+\gamma_{n}(P)\right)=\gamma_{n}(F)$ and $x+\gamma_{n}(P) \in \operatorname{ker} \phi_{n}$.

Since $\phi_{n}$ is an isomorphism then $\operatorname{ker} \phi_{n}=\gamma_{n}(P)$. Therefore $x+\gamma_{n}(P)=$ $\gamma_{n}(P)$, and $x \in \gamma_{n}(P)$. Consider $H \cap \gamma_{n}(P)=\gamma_{n}(H)$, then $x \in \gamma_{n}(H)$. Hence $\operatorname{ker} \varphi \subseteq \gamma_{n}(H)$.

Let $h$ be an element of $\gamma_{n}(H)$. If we consider $H \cap \gamma_{n}(P)=\gamma_{n}(H)$, then $h \in H$ and $h \in \gamma_{n}(P)$.

By definition of $\varphi, \varphi(h)=\phi(h)+\gamma_{n}(\phi(H))$ and so $\phi(h) \in \gamma_{n}(F)$.Now recall that $\gamma_{n}(\phi(H))=\phi(H) \cap \gamma_{n}(F), \phi(h) \in \phi(H)$ and $\phi(h) \in \gamma_{n}(F)$, then $\phi(h) \in \gamma_{n}(\phi(H))$. Hence, $\gamma_{n}(H) \subset \operatorname{ker} \varphi$, then we have

$$
\operatorname{ker} \varphi=\gamma_{n}(H)
$$

By the Isomorphism Theorem,

$$
H / \gamma_{n}(H) \cong K / \gamma_{n}(K)
$$

Thus the subalgebra $H$ is a parafree Lie algebra.

Theorem 7. The quotient algebra of a parafree Lie algebra is parafree.

Proof. Let $P$ be a parafree Lie algebra and $I$ be an ideal of $P$.

Let $u \in \bigcap_{n=1}^{\infty} \gamma_{n}(P / I)$. Then for every $n$

$$
u \in \gamma_{n}(P / I)=\left(\gamma_{n}(P)+I\right) / I
$$

Let $u=a+I$, where $a \in \gamma_{n}(P)+I$. It is clear that $a \in \bigcap_{n=1}^{\infty} \gamma_{n}(P)+I$. Since $P$ is residually nilpotent we get $a \in I$. Thus $u=I$ and $\bigcap_{n=1}^{\infty} \gamma_{n}(P / I)=0$

Hence $P / I$ is a residually nilpotent Lie algebra. Now we want to prove that $P / I$ has the same lower central sequence as a free Lie algebra. Consider $(P / I) / \gamma_{n}(P / I)$ : 
Using $\left.\left(\gamma_{n}(P)+I\right) / I\right) \cong \gamma_{n}(P) / I$.We obtain

$$
\begin{gathered}
(P / I) /\left(\gamma_{n}(P / I)\right) \cong(P / I) /\left(\left(\gamma_{n}(P)+I\right) / I\right) \\
\cong(P / I) /\left(\gamma_{n}(P) / I\right) \cong P / \gamma_{n}(P)
\end{gathered}
$$

This shows that $(P / I) /\left(\gamma_{n}(P / I)\right)$ has the same lower central sequence as $P$. Thus

$$
(P / I) / \gamma_{n}(P / I) \cong F / \gamma_{n}(F) .
$$

That is, the quotient algebra $P / I$ is parafree.

Lemma 8. Let $P$ be a parafree Lie algebra with finite rank. If $P$ and $P / I$ have the same rank, then $I=\{0\}$.

Proof. If $P$ and $P / I$ have the same rank, then for every positive integer $n$,

$$
P / I \cong(P / I) / \gamma_{n}(P / I) \cong\left((P / I) /\left(\gamma_{n}(P)+I\right) / I\right) \cong P / \gamma_{n}(P)+I .
$$

It is known that $P / I$ is finitely generated nilpotent Lie algebra, then by [11], $P / I$ is Hopfian. Furthermore,

$$
P /\left(\gamma_{n}(P)+I\right) \subseteq P / \gamma_{n}(P)
$$

and

$$
P / \gamma_{n}(P) \cong P /\left(\gamma_{n}(P)+I\right) .
$$

That is a contradiction since $P / \gamma_{n}(P)$ is Hopfian. Thus for all positive integers $n, I \subseteq \gamma_{n}(P)$, then $I=\{0\}$.

\section{References}

[1] Yu.A. Bahturin, Identical relations in Lie algebras, VNU Science Press b.v., Utrecht (1987).

[2] G. Baumslag, Groups with the same lower central sequence as a relatively free group I. The groups, Trans. Amer. Math. Soc., 129 (1967), 308-321.

[3] G. Baumslag, Groups with the same lower central sequence as a relatively free group. II Properties, Trans. Amer. Math. Soc., 142 (1969), 507-538.

[4] G. Baumslag, Parafree groups, Progress in Math., 248 (2005), 1-14. 
[5] G. Baumslag, U. Stammbach, On the inverse limit of free nilpotent groups, Comment. Math. Helvetici, 52 (1977), 219-233.

[6] G. Baumslag, S. Cleary, Parafree one-relator groups, J. Of groups theory (2006), 191-201.

[7] G. Baumslag, S. Cleary, G. Havas, Experimenting with infinite group, Experimental Math., 13 (2004), 495-502.

[8] H. Baur, Parafreie Liealgebren und homologie, Diss. Eth Nr., 6126 (1978).

[9] H. Baur, A note on parafree Lie algebras, Commun. in Alg., 8, No. 10 (1980), 953-960.

[10] Margaret H. Dean, Finitely Generated Metbalian and Solvable Groups, Ph.D. Thesis, City University of New York (2008).

[11] T. Evans, Finitely presented loops, lattices, etc. are Hopfian, J. London Math. Soc., 44 (1969), 551-552.

[12] M.A. Knus, U. Stammbach, Anwendungen der homologietheorie der Liealgebren auf zentralreihren unda uf prasentierungen, Comment. Math. Helvetici, 42 (1967), 297-306. 